\title{
Gerhard Gottschalk
}

\section{Nachruf auf Hans Günter Schlegel \\ 24. Oktober 1924 - 22. März 2013}

Herr Präsident, meine Damen und Herren,

Im Januar 1960 begegnete ich Hans Günter Schlegel zum ersten Mal. Ich besuchte ihn in dem ehemaligen Gebäude des Instituts für Mikrobiologie in der Goßlerstraße 16. Dieses Gebäude, um 1900 herum errichtet, beherbergte das in Deutschland erste naturwissenschaftlich ausgerichtete mikrobiologische Institut. Nach Alfred Koch und August Rippel-Baldes war Hans Günter Schlegel der dritte Lehrstuhlinhaber. Als ich mich im Eingangsbereich des Instituts umschaute, stürmte ein jugendlich wirkender Mann mit wehendem Kittel die Treppe herunter und verschwand hinter einer Labortür. Als er wieder herauskam, war es schwierig ihn zu stoppen und anzusprechen. Dann aber hatten wir in aller Ruhe ein längeres Gespräch, in dem ich seine Begeisterung für die Bakterien und ihre Rolle in der Natur spürte, eine Begeisterung, die im wahrsten Sinne des Wortes ansteckend war und die er sich bis ins hohe Alter erhielt.

Hans Günter Schlegel wurde am 24. Oktober 1924 in Leipzig geboren. Sein Vater war Lehrer, der seinen Sohn schon früh an die Arbeiten im häuslichen Garten heranführte und in ihm die Liebe zur Welt der Pflanzen weckte. Der Bildungsweg schien vorgezeichnet, Gymnasium und Studium der Naturwissenschaften, endete dann aber vorerst nach elfjähriger Schulzeit mit dem Notabitur und dem Kriegsdienst bei der Luftwaffe. Aus britischer Gefangenschaft im Mai 1945 entlassen, besorgte sich Hans Günter Schlegel ein Fahrrad und radelte, wie er mir einmal erzählte, so schnell es ging, vom Niederrhein zurück nach Leipzig. Er gehörte zu den Glücklichen, seine Eltern und seine Schwester waren wohlauf und das Elternhaus war unversehrt. Sofort machte er da weiter, wo er 1942 aufgehört hatte: Abitur für Kriegsteilnehmer und danach Immatrikulation für Naturwissenschaften an der Universität Leipzig, nach zwei Jahren Wechsel an die Universität Halle, und dort wurde er 1950 promoviert.

In diesen wenigen Jahren muss sich in ihm eine Wandlung vollzogen haben. Neben seiner Bewunderung der sichtbaren Natur, entwickelte sich ein starkes Interesse an dem Unsichtbaren, an den Bakterien. Sie faszinierten ihn mehr und mehr, besonders ihr Stoffwechselgeschehen, wobei es in seinen Forschungsarbeiten anfangs durchaus noch farbig zuging. Sein Doktorvater Johannes Buder beschäftigte sich mit Purpurbakterien, die sich in den Parkteichen des ehemaligen Ritterguts in Ostrau prächtig vermehrten und unter der Oberfläche purpurne Teppiche bildeten. Man konnte sie mit einer Kelle abschöpfen und dann 
im Labor untersuchen, beispielsweise ihr phototaktisches Verhalten beobachten. Die Lichtreizreaktionen standen dann auch im Mittelpunkt der Dissertation von Hans Günter Schlegel. Besonders prägend für ihn war seine Zusammenarbeit mit Kurth Mothes, einer Persönlichkeit von großer Ausstrahlung und großem Einfluss, nicht zuletzt als langjähriger Präsident der Deutschen Akademie der Naturforscher, Leopoldina. In seinem Arbeitskreis, im Institut für Kulturpflanzenforschung in Gatersleben bearbeitete Schlegel ab 1952 die bis dahin wenig untersuchten sogenannten Knallgasbakterien. Diese leben davon, Wasserstoff und Sauerstoff zu Wasser umzusetzen ohne dass es knallt, die freiwerdende Energie in Stoffwechselenergie umzuformen und damit aus Kohlendioxid, Wasser und Mineralien Zellsubstanz zu produzieren. Was für ein aufregendes Leben! Kein höherer Organismus ist dazu in der Lage.

Mit seinen Kenntnissen und Erfahrungen im Gepäck ging Hans Günter Schlegel auf eine für einen DDR-Bürger ungewöhnliche Wanderschaft. Sie war nur mit einer so einflussreichen Persönlichkeit wie Kurth Mothes im Hintergrund möglich. Das Jahr 1956 verbrachte er als Stipendiat im Biochemischen Institut der Ludwig-Maximilians-Universität in München und erwarb sich schnell die Anerkennung des dortigen Chefs und späteren Nobelpreisträgers Feodor Lynen. Ein Enzym, eine spezielle Carboxylase, beteiligt am Leucinabbau in der Leber, wurde benötigt. Hans Günter Schlegel gewann dieses Enzym nicht aus der Leber, in der es nur in geringer Menge vorkommt, sondern aus Bakterien, die er aus dem Wurzelbereich von Baldrianpflanzen isoliert hatte und die viel von diesem Enzym bildeten. Diese Vorgehensweise beeindruckte die Biochemiker.

Im Münchener Institut gingen ausländische Gastwissenschaftler ein und aus, darunter Lester Krampitz von der Case Western Reserve University in Cleveland, Ohio (USA). Mit ihm freundete sich Hans Günter Schlegel an, sie kooperierten und Krampitz bot ihm eine Postdoc-Stelle an. So arbeitete H. G. Schlegel ab 1957 (wohl noch als DDR-Bürger) in den USA und dort häuften sich glückliche Umstände. Er war wissenschaftlich erfolgreich, heiratete seine Frau Ingeborg, sein Sohn Peter wurde geboren (die Töchter Dagmar und Uta folgten in Deutschland), und er erhielt den Ruf auf den Lehrstuhl für Mikrobiologie an der Universität Göttingen, den er annahm. So wurde ein Kreis geschlossen, jedoch nicht ganz, denn zwischen Göttingen und Halle verlief die deutsch-deutsche Grenze.

Schlegels Wirken werde ich in vier Abschnitten umreißen. H. G. Schlegel war ein Motor bei der Etablierung des Faches Mikrobiologie in Deutschland. In Göttingen fand er 1958 ein kleines, leistungsfähiges Institut vor, das sich auf dem Gebiet der Bodenmikrobiologie einen Namen gemacht hatte. Die vorhandenen Stärken nutzend entwickelte er dieses Institut zu einem Zentrum für naturwissenschaftliche Mikrobiologie mit großer nationaler und internationaler Ausstrahlung. Dafür war zunächst ein räumlicher und personeller Ausbau erforderlich. Aus dem klei- 
nen Backsteinbau in der Goßlerstraße wurde ein geräumiges Gebäude im Nordbereich der Universität mit hervorragenden Möglichkeiten für die Forschung und die Lehre; aus einer Abteilung wurden im Laufe der Jahre fünf Abteilungen und die Forschungsgebiete reichten von der Physiologie, Biochemie und Mikromorphologie der Mikroorganismen bis zur Biotechnologie. Schlegel entwickelte Visionen, die Mitarbeiterinnen und Mitarbeiter sowie die Studierenden in ihren Bann zogen. Hinreißend konnte er über die Bedeutung Wasserstoff oxidierender Bakterien, über Purpurbakterien und die Mutationsforschung sprechen. Seinem „mikrobiologischen“ Charme konnten sich auch Politiker und Ministerialbeamte nicht entziehen. Und so erreichte er zusammen mit Otto Kandler (München) 1969 auch die Gründung der „Deutschen Sammlung von Mikroorganismen“ in Göttingen, die später als eine Einrichtung der Leibniz-Gemeinschaft nach Braunschweig umzog.

Von der Entwicklung der Göttinger Mikrobiologie ging ein Signal aus, das zur Etablierung dieses Faches an einer Reihe von Universitäten in der Bundesrepublik führte. Hierzu trug wesentlich Schlegels fundamentaler Beitrag als Buchautor bei. Es gab in den 1960er Jahren kein Lehrbuch, in dem die Physiologie, der Stoffwechsel und die Genetik der Bakterien für den Lehrbetrieb an Universitäten beschrieben waren. Entsprechend groß war der Erfolg von Schlegels „Allgemeine Mikrobiologie“, die 1969 erschien. Bis 1992 betreute er sechs weitere Auflagen seines Werkes. Bis heute hat kein deutschsprachiges Lehrbuch einen vergleichbaren Einfluss auf die Ausbildung und auf die Verbreitung von Kenntnissen über Mikroorganismen gehabt. Wie groß auch in anderen Sprachräumen die Lücke war, die dieses Buch füllte, dokumentiert die Übersetzung in sieben Sprachen, ins Russische, Englische, Spanische, Polnische, Persische, Indonesische und Italienische. Man kann schon sagen, es war ein Welterfolg, der Generationen von Studentinnen und Studenten die unsichtbare Welt der Mikroben erschloss.

Neben einigen Monographien, auf die noch eingegangen wird, ist Schlegels „Geschichte der Mikrobiologie“ ein weiteres beachtenswertes Buch; er schrieb es als Emeritus. Anhand der Biographien von Entdeckern, von Antonie van Leeuwenhoek, verstorben 1723, bis Harland Wood, verstorben 1991, wird die Entwicklung der Mikrobiologie dargestellt. Dieses Buch ist eine Fundgrube für den historisch interessierten Naturwissenschaftler. Hier folgt Schlegel der Empfehlung von Theodor Heuss „die Naturwissenschaftler und die Ärzte sollen anfangen, die Geschichte der Naturwissenschaften und der Medizin selber zu studieren“ (1950).

H. G. Schlegels Forschungsinteressen werden durch einige Monographien dokumentiert, die er als alleiniger Autor bzw. zusammen mit Coautoren verfasste: „Anreicherungskultur und Mutantenauslese“, „Microbial Production and Utilization of Gases“, „Microbial Energy Conversion“, „Hydrogenases“, „Autotrophic Bacteria“ und „Bacterial Polyhydroxy Alcanoates“. Die mikrobielle Umset- 
zung von Wasserstoff lag ihm besonders am Herzen; die vielen Bakterienarten, die unter verschiedensten Bedingungen die bereits erwähnte Knallgasreaktion durchführen. Die Funktionsweise des sehr komplex aufgebauten Enzyms Hydrogenase, das den Wasserstoff aufnimmt und in den Stoffwechsel einführt, wurde eingehend untersucht. Eine Bakterienart stand dabei für ihn im Mittelpunkt: Ralstonia eutropha, und es war für ihn eine besondere Freude, dass die vollständige Sequenz des für Bakterienverhältnisse großen Genoms dieser Mikrobe zu seinem 80. Geburtstag präsentiert werden konnte. $R$. eutropha ist auch die Quelle der Poly- $\beta$-Hydroxyalkanoate und der zuständigen Gene. Anstelle von Kunststoffen finden diese biologisch abbaubaren Biopolymere langsam ihren Weg in die Anwendung. Ihre Entwicklung begann in Göttingen und führte u.a. zur Verleihung des Philip-Morris-Preises für Zukunftstechnologien an Schlegel und Kollegen im Jahre 1992. Auch die Kohlenmonoxidverwertung interessierte ihn. Für manche Bakterienarten ist $\mathrm{CO}$ so etwas Ähnliches wie $\mathrm{H}_{2}$. Sie oxidieren es $\mathrm{zu} \mathrm{CO}_{2}$ und verwerten die freiwerdenden Elektronen für die Bildung von Stoffwechselenergie. Diese carboxidophilen Organismen können wahlweise CO oder $\mathrm{H}_{2}$ umsetzen und sorgen dafür, dass sich das giftige CO nicht im Boden anhäuft.

Lebhaftes Interesse hatte Schlegel an der Isolierung von Mutanten und ihren gezielten Einsatz bei der Untersuchung von Stoffwechselprozessen. Dieses führte ihn unter anderem zur Schwermetallresistenz bei Bakterien, für die die notwendige genetische Information häufig auf extrachromosomaler DNA, auf so genannten Plasmiden, liegt. Besonders hatte es ihm die Nickelresistenz angetan. 1989 reiste Schlegel nach Neukaledonien, einer Insel bestehend aus stark manganund nickelhaltigem Gestein. Die Blätter des dort wachsenden Nickelbaumes, Sebertia acuminata, enthalten mehr als $1 \%$ Nickel. Der blaugrüne Latex des Baumes hat einen Nickelgehalt von $25 \%$. Schlegel interessierte sich natürlich für die Bakterien in den Böden unter diesen Bäumen. Sie waren resistent gegenüber Lösungen mit sage und schreibe 1,5 g Nickel pro Liter, was Ausdruck einer nahezu unglaublichen Anpassung ist. Der Mechanismus ist simpel: Die Bakterien pumpen nickelhaltige Lösungen genauso schnell aus dem Zellinneren nach draußen wie sie eindringen. Ein breites Band von wissenschaftlichen Interessen und eine nie versiegende Begeisterung, die sich von Purpur- über Knallgasbakterien und Mutationsforschung bis hin zur Nickelresistenz, aber eigentlich über die gesamte Mikrobiologie erstreckte, zogen sich durch das überaus erfolgreiche Forscherleben von Hans Günter Schlegel. Zahlreiche Ehrungen und Ehrenämter stellten sich ein.

Schlegel war dreifacher Ehrendoktor, unter anderem seiner Alma Mater in Halle, Ehrenmitglied mehrerer wissenschaftlicher Gesellschaften und Mitglied von vier Wissenschaftsakademien. Besonders eng fühlte er sich der Deutschen Akademie der Naturforscher, Leopoldina, jetzt Nationale Akademie, verbunden. 
Schließlich hatte er in den 1950er Jahren mit Kurth Mothes, einem ihrer herausragenden Präsidenten, zusammengearbeitet. Regelmäßig fuhr er von Göttingen aus zu den Jahresversammlungen nach Halle und lud junge Mitarbeiterinnen und Mitarbeiter zur Teilnahme an den Sitzungen ein. So gehörte er zu den Wissenschaftlern, die während der langjährigen Teilung Deutschlands durch ihr Engagement und ihre Hilfsbereitschaft Bindeglieder zwischen Ost und West waren. In diesem Zusammenhang ist auch die Verleihung der Cothenius-Medaille der Leopoldina (2005) an Schlegel zu sehen.

In die Göttinger Akademie wurde Schlegel 1965 gewählt. Groß war sein Interesse an den Veranstaltungen unserer Akademie, und so war es naheliegend, dass bald größere Aufgaben in der Akademie auf ihn zukamen. Er wurde 1984 zum Vizepräsidenten gewählt und war von 1986 bis 1988 ihr Präsident. Vor 28 Jahren war die Akademienlandschaft eine andere als heute. Außendarstellung und Vernetzung waren weit weniger ausgeprägt. Es gab die Konferenz der Wissenschaftsakademien, aus der später die Union der Wissenschaftsakademien hervorging. Die Konferenz bestand aus fünf Mitgliedern. Neben Göttingen waren es die Akademien mit Sitz in München, Heidelberg, Mainz und Düsseldorf. Erst nach der Wiedervereinigung kamen die Sächsische und die Berlin-Brandenburgische Akademie sowie die 2004 gegründete Hamburger Akademie hinzu. Das wissenschaftliche Leben in unserer Akademie wurde durch die Sitzungen und besonders durch die wissenschaftlichen Unternehmungen bestimmt. Immerhin waren es während der Präsidentschaft von Schlegel 18 Vorhaben aus dem Akademienprogramm und etwa 15 weitere Vorhaben, die von Mitgliedern unserer Akademie zusammen mit Fachkollegen durchgeführt wurden. Darunter waren sieben naturwissenschaftliche Projekte. Die Betreuung dieser Vorhaben und die Sicherung ihrer Finanzierung lag Schlegel besonders am Herzen und so steht er mit seinem Wirken in einer Reihe mit seinen bedeutenden Vorgängern und Nachfolgern. Hans Günter Schlegel hat sich um unsere Akademie verdient gemacht, die Akademie wird ihm ein ehrendes Andenken bewahren. 